Moderne Therapie des Mammakarzinoms

\title{
Paradigmenwechsel hin zu einer chronischen Behandlung
}

Es gibt Hinweise darauf, dass Brustkrebs durch moderne Behandlungsmöglichkeiten immer mehr zu einer chronischen Erkrankung wird. Seit mehr als 10 Jahren steht mit Letrozol (Femara $\left.{ }^{\circledR}\right)$ ein effektiver Aromatasehemmer zur Verfügung, der die periphere und intratumorale Östrogensynthese effektiv hemmt. Bei postmenopausalen Frauen mit einem hormonrezeptorpositiven primären Mammakarzinom führt die adjuvante «Upfront-Therapie» mit Letrozol - im Vergleich zur Tamoxifenbehandlung - zu einer signifikanten Reduktion von frühen Fernmetastasen und Rezidiven.

Im Rahmen der antineoplastischen Tumortherapie des Mammakarzinoms kann es durch die vielfach eingesetzten Anthrazykline zu unvorhersagbaren und irreversiblen kardialen $\mathrm{Ne}$ benwirkungen kommen, die sich durch die Gabe des Kardioprotektivums Dexrazoxan (Cardioxane $^{\circledR}$ ) reduzieren lassen, ohne dass eine Wirksamkeitsminderung der Anthrazykline befürchtet werden muss.

«Auch nach der Entfernung des hormonsensiblen Mammakarzinoms bleibt ein erhebliches Rückfallrisiko bestehen», erklärte Prof. Dr. med. Fritz Jänicke vom Universitätsklinikum Hamburg-Eppendorf. «Daher ist heute eine adjuvante endokrine Therapie selbstverständlich», so der Experte. Nach den Erfahrungen der letzten Jahre, basierend auf verschiedenen klinischen Studien, werde heute vorwiegend eine «Upfront-Therapie» mit Aromatasehemmern wie Letrozol eingesetzt nach dem Motto: «Hochwirksam von Anfang an!»

\section{Weniger Frührezidive und Fernmetasta- sen bei adjuvanter "Upfront-Therapie" mit Letrozol}

Als Gründe für einen «Upfront-Einsatz» nannte der Hamburger Gynäkologe die Beobachtung, dass das Rückfallrisiko schon kurz nach der Operation deutlich ansteigt und bereits um das zweite postoperative Jahr die Höchstwerte erreicht werden (Doughty et al., 2007). Frühe Fernmetastasen bilden dabei mit über $70 \%$ den Hauptanteil aller Rezidive beim hormonrezeptorpositiven Mammakarzinom. Deutlich seltener kommt es zu lokoregionalen (etwa 20\%) oder kontralateralen Rezidiven (etwa 10\%). Fernmetastasen sind vor allem für den Rezidiv-Peak nach 2 Jahren verantwortlich. Etwa $78 \%$ aller Patientinnen mit Fernmetastasen und nur 24\% derer mit Lokalrezidiven sterben nach 5 Jahren.

Für Jänicke ist die «bestmögliche Wirksamkeit der adjuvanten Behandlung gegen frühe Fernmetastasen» von zentraler Bedeutung für die Langzeitprognose der Brustkrebspatientinnen. In der vierarmigen BIG 1-98-Studie (Coates et al., 2007) wurde nachgewiesen, dass es bei einer medianen Nachbeobachtungszeit von 51 Monaten unter Letrozol im Vergleich zu Tamoxifen zu einer signifikanten Reduktion des Fernmetastasenrisikos $(19 \%, p=0,03)$ kam.

Außerdem konnte eine - im Vergleich zur Tamoxifenbehandlung - bessere Wirksamkeit hinsichtlich des krankheitsfreien Überlebens dokumentiert werden (Abb. 1). Nach einer Schätzung von Jänicke können in Deutschland jährlich mehr als 1000 Rezidive und Metastasen verhindert werden, wenn die Patientinnen eine «Upfront-Behandlung» mit einem Aromatasehemmer erhalten.

Der Experte fasste die Ergebnisse der BIG 1-98-Studie wie folgt zusammen: «Letrozol ist wirksamer als Tamoxifen in der Prävention früher Rezidive. Dies gilt besonders auch für die folgenden Subgruppen, die ein erhöhtes Risiko haben: Patientinnen mit Tumoren mit mehr als vier befallenen Lymphknoten und mit Tumoren, die größer als $2 \mathrm{~cm}$ sind. Auch diese Patientinnen profitieren von einer initialen Letrozoltherapie!»

Prof. Dr. med. Klaus Höffken vom Universitätsklinikum Jena ergänzte, dass Letrozol in vitro und in vivo der stärkste Aromatasehemmer ist. Er betonte, dass sich der Aromatase- hemmer im Rahmen klinischer Studien in allen Settings bewährt hat: adjuvant «Upfront», second-line und first-line. Zusätzlich ist er als einziger seiner Wirkstoffklasse in der erweiterten adjuvanten Therapie nach 5 Jahren Tamoxifen zugelassen und erreichte hier bei nodalpositiven Mammakarzinompatientinnen einen Gesamtüberlebensvorteil.

\section{Anthrazykline sind kardiotoxisch}

Viele der häufig eingesetzten Zytostatika wie z.B. die Anthrazykline Doxorubicin, Daunorubicin, Epirubicin und Idarubicin führen zu kardialen Nebenwirkungen. Für PD Dr. med. Thomas M. Suter vom Universitätsspital Bern sind die Anthrazykline ein ernstes Problem, denn sie können bei der Langzeitbehandlung und bei der Gabe hoher Dosen eine irreversible Herzinsuffizienz bewirken, die dann zum Herzversagen führen kann.

Auch einige der neuen Brustkrebstherapeutika wie z.B. monoklonale Antikörper (Trastuzumab) oder Substanzen aus der Gruppe der Tyrosinkinasehemmer weisen ein kardiales Risikopotenzial auf. Das bedeutet, dass bereits vorgeschädigten Patientinnen diese neuen Onkologika nicht mehr verabreicht werden können.

Die kardiotoxische Wirkung der Anthrazykline beschreibt der Schweizer Kardiologe wie folgt: Sie bewirken zunächst in den Herzmuskelzellen einen oxidativen Stress, der dann zu einer myofibrillären Desorganisation führt, gefolgt von Apoptose und Nekrose der Myozyten. Diese kardialen Effekte sind umso schwieriger zu therapieren, je weiter die Schädigung vorangeschritten ist.

Aus der Sicht der Krebspatientin wäre es wünschenswert, das Herz frühzeitig und effektiv vor den kardiotoxischen Wirkungen der Anthrazykline zu schützen. Denn die Kardiotoxizität bedeutet für den Kostenträger einen hohen Kostenaufwand. Vor jedem neuen Therapiezy-

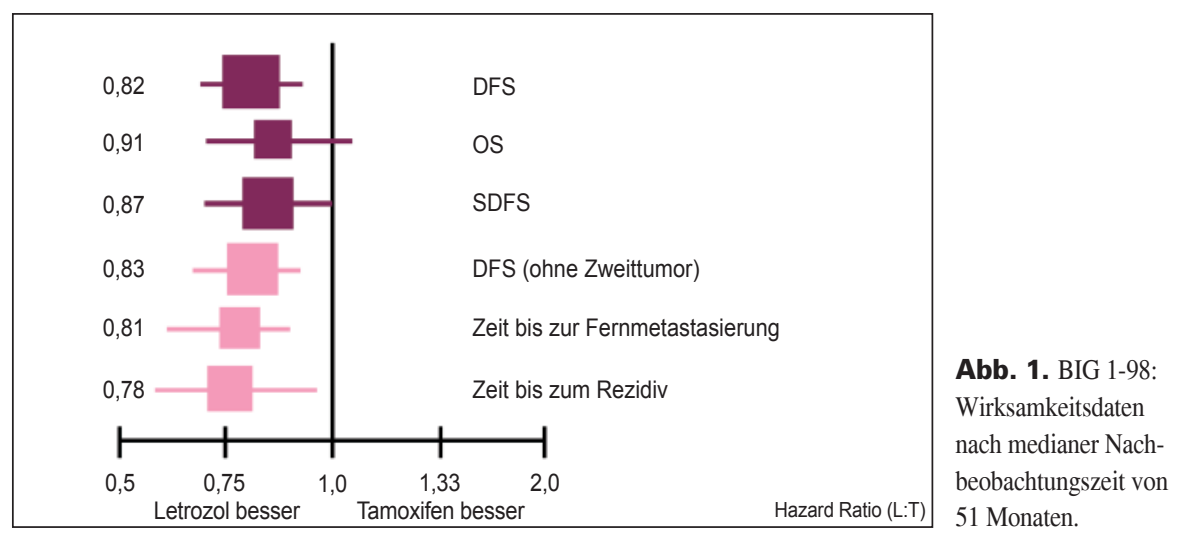




\section{Innovationen • Innovations}

Abb. 2. Durchschnittliche Reduktion der linksventrikulären Ejektionsfraktion (LVEF) nach kumulativen Doxorubicin-Dosen von $150 \mathrm{mg} / \mathrm{m}^{2}$ bis 600 $\mathrm{mg} / \mathrm{m}^{2}$ : Vergleich mit und ohne begleitende Dexrazoxan-Gabe.

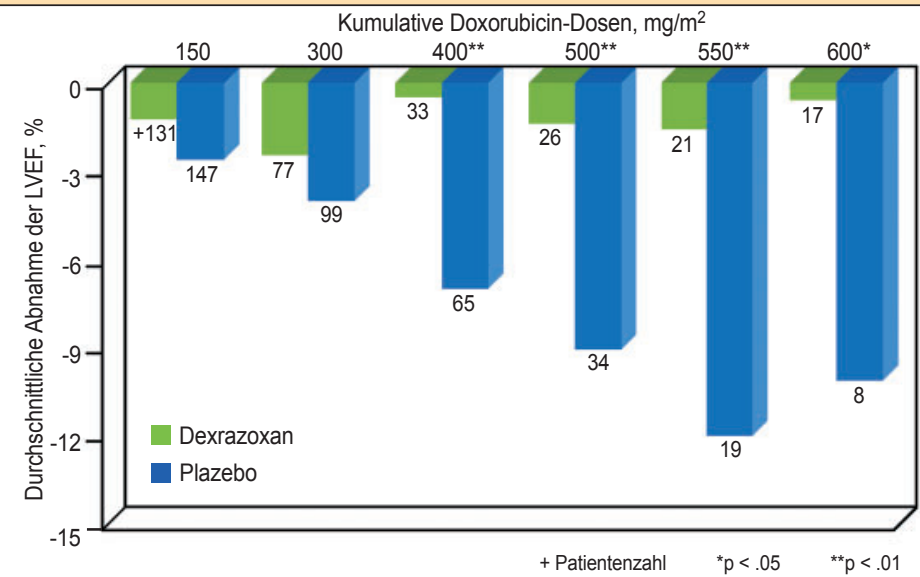

klus muss ein Elektrokardiogramm erstellt werden. Außerdem muss das Herz in regelmäBigen Abständen echokardiographisch untersucht werden.

\section{Anthrazyklin-induzierte Kardio- toxizität wird reduziert}

Doch vor Herzschäden können Patienten durch Gabe eines Kardioprotektivums geschützt werden, so Suter, da dies die Bildung der toxischen Anthrazyklin-Eisen-Komplexe verhindert. Das Kardioprotektivum Dexrazoxan $\left(\right.$ Cardioxane $\left.^{\circledR}\right)$ ist seit Juni 2007 in Deutschland zugelassen zur Vorbeugung der chronischen kumulativen Kardiotoxizität bei Patienten mit einer fortgeschrittenen und/oder metastasierten Krebserkrankung, die mit Doxorubicin oder Epirubicin behandelt werden. Im Oktober 2007 hat die Kommission Mamma der Arbeitsgemeinschaft Gynäkologische Onkologie (AGO) Dexrazoxan in seine aktuelle Leitlinie aufgenommen und empfiehlt den Einsatz bei kardialen Risikopatientinnen.

Dexrazoxan schützt das Herz, ohne die klinische Antitumor-Effektivität der Anthrazyklin-haltigen Chemotherapie negativ zu beein- trächtigen, betonte Prof. Dr. med. Sandra M. Swain vom Washington Cancer Institute in Washington, USA. Dexrazoxan erhöhe auch nicht die Rate der für die Anthrazyklintherapie bekannten nicht-kardialen Nebenwirkungen. In einer retrospektiven Analyse von drei Studien zur Häufigkeit der Herzinsuffizienz bei Frauen, die mit dem Anthrazyklin Doxorubicin behandelt worden waren, konnte sie zeigen, dass mit steigender Doxorubicin-Dosis die linksventrikuläre Ejektionsfraktion (LVEF) in der Plazebogruppe immer geringer wurde, während in der Verumgruppe die LVEF nahezu unverändert blieb (Swain et al., 2003). Bei Dosen von mehr als $400 \mathrm{mg} / \mathrm{m}^{2}$ waren die Unterschiede zwischen Verum- und Plazebogruppe statistisch signifikant.

\section{Dr. Richard Roth, Kirchheim}

\section{Quelle}

Satelliten-Symposium «Brustkrebs: Paradigmenwechsel hin zu einer chronischen Behandlung» anlässlich des Deutschen Krebskongresses (DKK), Berlin, 22. Februar 2008.
Bisphosphonat Zoledronat schützt vor Knochenkomplikationen

Knochenmetastasen sind schwerwiegende Komplikationen bei zahlreichen Tumorerkrankungen. So sind zwischen 65 und 75\% der Mamma- und der Prostatakarzinompatienten und fast alle Patienten mit einem Multiplen Myelom im Verlaufe ihrer Erkrankung von ossären Metastasen betroffen. Werden diese nicht rechtzeitig adäquat behandelt, kann es zu Frakturen, Spinalkompressionen und einer tumorinduzierten Hyperkalzämie kommen, die zu starken Knochenschmerzen und lebensgefährlichen Komplikationen führen.

Das intravenöse Bisphosphonat Zoledronat (Zometa $\left.{ }^{\circledR}\right)$ ist zugelassen zur Behandlung von Knochenmetastasen bei allen Tumorentitäten, zur Prävention skelettbezogener Komplikationen bei fortgeschrittenen, auf das Skelett ausgedehnten Tumorerkrankungen und auch zur Therapie der tumorinduzierten Hyperkalzämie (TIH). Zoledronat hat sich in verschiedenen klinischen Studien als besonders wirksam erwiesen, da es die Entwicklung von Knochenkomplikationen verzögert und deren Häufigkeit reduziert. Das Präparat ist das einzige Bisphosphonat, welches nicht nur mit Plazebo, sondern auch mit dem Standard-Bisphosphonat Pamidronat verglichen wurde: Zoledronat reduzierte bei Patienten mit Mammakarzinom und Multiplem Myelom das relative Risiko für eine erste Knochenkomplikation gegenüber Pamidronat um insgesamt 20\%. Kam es zu einer Knochenkomplikation (SRE), trat diese unter der Behandlung mit Zoledronat im Median signifikant später auf (310 vs. 174 Tage) (Abb. 3).

Die Behandlung mit Zoledronat führt auch zu einer deutlichen Reduktion der tumorbedingten Knochenschmerzen, was sowohl die Mobilität als auch die Lebensqualität der Patienten deutlich verbessert. Prof. Dr. med. Tanja Fehm, Tübingen, stellte fest: «Moderne Bisphosphonate tragen dazu bei, die Lebensqualität und die funktionelle Unabhängigkeit unserer Patientinnen mit einem fortgeschrittenen Brustkrebs zu bewahren.» Zudem sprach sie die Empfehlung aus, möglichst früh mit einem hoch wirksamen Bisphosphonat wie Zoledronat zu intervenieren.

PD Dr. med. Wolfgang Janni, München, forderte, dass bei Brustkrebspatientinnen, die ein erhöhtes Osteoporoserisiko aufweisen, der Knochenschutz in das Gesamttherapiemanagement eingebunden werden sollte: "Die therapieassoziierte Osteoporose von Brustkrebspatientinnen lässt sich heute effizient mit Bisphosphonaten behandeln.» Auch die American Society of Clinical Oncology empfehle den Einsatz von Bisphosphonaten zur Verhinderung des Knochendichteverlustes bei Frauen, die eine Aromatasehemmertherapie erhalten.
Abb. 3. Die erste Knochenkomplikation tritt bei Mammakarzinompatientinnen mit mindestens einer osteolytischen Läsion unter Pamidronat im Median nach 174 Tagen auf, unter Zoledronat erst nach 310 Tagen.

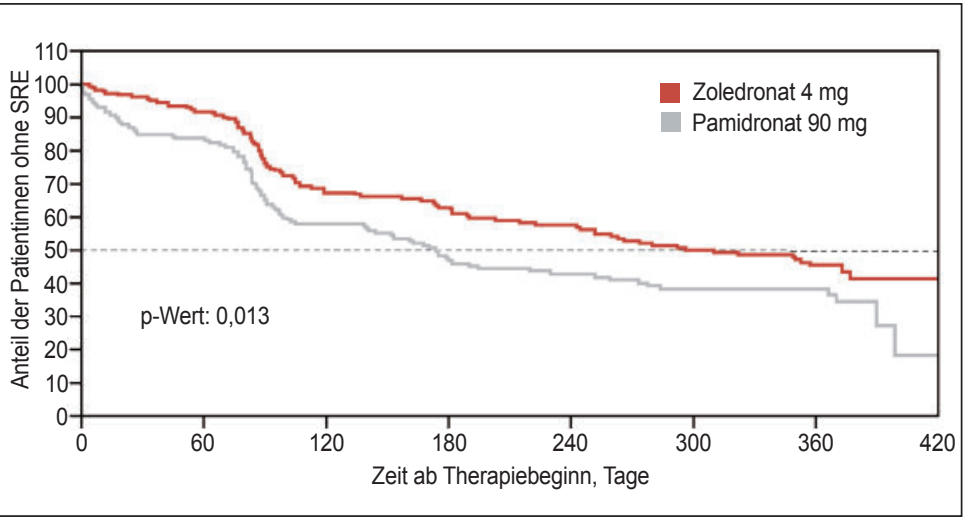

\section{Impressum \\ Moderne Therapie des Mammakarzinoms \\ Paradigmenwechsel hin zu einer chronischen Behandlung \\ Innovationen in BREAST CARE 3 | 2 | 08 \\ (c) 2008 by S. Karger Verlag für Medizin und Naturwissenschaften $\mathrm{GmbH}$ \\ Lörracher Straße 16a \\ 79115 Freiburg, Deutschland}

Mit freundlicher Unterstützung de Novartis Pharma GmbH, Nürnberg.

Der Verlag und die Herausgeber der Zeitschrift übernehmen keine Verantwortung für diese Rubrik. 